\title{
Resilience in aging and age-related disease. Abstracts from the 2019 Barshop Conference on Aging
}

\author{
Editors: Warren Ladiges, Yuji Ikeno, and Liang Wang
}

\begin{abstract}
Resilience in Aging and Age-related Disease was the topic of the 2019 Barshop Conference on Aging held at the Mayan Dude Ranch in Bandera, TX. The conference was sponsored by the Barshop Institute for Longevity and Aging Studies at the University of Texas Health San Antonio, San Antonio, TX. The conference organizers were Sara Espinoza, MD, MSc, Adam Salmon, PhD, and Amrita Kamut, PhD. Abstracts from the conference are published in thematic format as 1) Pharmacological Targets for Resilience, 2) Exercise Training and Resilience, 3) Cellular Stress Resilience, 4) Nonhuman Primate Models of Resilience, and 5) Novel Procedures to Support Resilience Studies.
\end{abstract}

Keywords: Resilience to aging, stress tests, biomarkers, senescence, age-related disease, enhancing resilience, animal models of resilience

\section{Introduction}

The 2019 Barshop Conference on Aging was held at the Mayan Dude Ranch in Bandera, TX with the theme "Resilience in Aging and Age-related Disease". The conference was sponsored by the Barshop Institute for Longevity and Aging Studies at the University of Texas Health San Antonio, San Antonio, TX, and organized by Sara Espinoza, MD, MSc, Adam Salmon, PhD, and Amrita Kamut, PhD. Presentations consisted of platform sessions and posters.

The first platform session presented an Overview of Resilience: Defining and Predicting. The speakers were Dr. Francesca Maccharini, National Institute on Aging (NIA's view on resilience), Dr. Jeremy Watson, Johns Hopkins University School of Medicine (Physical frailty in older adults: Causes and interventions), and Dr. Cathleen ColonEmeric, Duke University School of Medicine (Predicting physical resilience in older adults).

The second session was on Identifying Biomarkers for Resilience. The speakers were Dr. Derek Huffman, Albert Einstein College of Medicine (Developing dynamic assays to quantify resilience in mice), Dr. Mini Jacob, University of Texas Health San Antonio (Gait: A biomarker of resilience), and Dr. Jamie Justice, Wake Forest University School of Medicine (Biomarkers of aging for geroscienceguided clinical trials).

The third session was on Cell Senescence in Resilience. The speakers were Dr. James Kirkland, Mayo Clinic (Aging, senolytics, age-related disease, and resilience), Dr. Mitzi Gonzales, University of Texas Health San Antonio
(Pilot investigation of senolytic therapy in Alzheimer's disease), and Dr. Nathan LeBrasseur, Mayo Clinic (Ageassociated changes in measures of physical resilience in mice).

The fourth session was on Intervention for Resilience. Can Resilience be Modified? The speakers were Dr. George Kuchel, University of Connecticut Center on Aging (Immune responses to vaccination in aging: Translational insights into immune resilience), Dr. Monica Serra, University of Texas Health San Antonio (Adjusting after stroke: Lessons to promote resilience during recovery), Dr. James White, Duke University School of Medicine (Chronic exposure to youthful circulation improves resilience in old mice), Dr. Alessandra Bartolomucci, University of Minnesota (Social stress drives senescence), and Dr. Arlan Richardson, University of Oklahoma Health Science Center (The effect of age and dietary restriction on resilience in mice).

The fifth and final session was on Big Data Biology to Address Resilience. The speakers were Dr. Warren Ladiges, University of Washington School of Medicine (Physical resilience is a predictor of healthy aging in mice), Dr. Ravi Varadhan, Johns Hopkins University School of Medicine (Dynamical systems thinking for studying resilience to clinical stressors), Dr. Sudha Seshadri, University of Texas Health San Antonio (Biology of dementias: Insights from fenetics, omics, and systems biology approaches), and Dr. Jamie Walker, University of Texas Health San Antonio (Primary age-related tauopathy (PART) and successful aging: Resistance and resilience to neuropathologic changes and cognitive decline in aging). 


\section{Poster abstracts}

\section{Pharmacological Targets for Resilience}

Evaluating novel microbiome-targeted treatments of insulin resistance

Eric Baeuerle $^{\mathrm{a} *}$, Hanyu Liang ${ }^{\mathrm{a}, \mathrm{b}}$, Vinutha Ganapathy ${ }^{\mathrm{a}, \mathrm{b}}$, Roman Fernan$\mathrm{dez}^{\mathrm{c}}$, Nattapol Sathavarodom ${ }^{\mathrm{a}, \mathrm{b}}$, Chen-Pin Wang ${ }^{\mathrm{c}}$, Sara Espinoza ${ }^{\mathrm{a}, \mathrm{b}}$, Nicolas Musi ${ }^{\mathrm{a}, \mathrm{b}}$

${ }^{a}$ Barshop Institute for Longevity and Aging Studies, University of Texas Health Science Center at San Antonio, TX, United States of America.

${ }^{\mathrm{b}}$ Geriatric Research Education and Clinical Center, Audie L. Murphy VA Medical Center, San Antonio, Texas, United States of America.

${ }^{c}$ Department of Epidemiology and Biostatistics, University of Texas Health Science Center at San Antonio, TX Center, San Antonio, Texas, United States of America.

The human gut and its microbial contents (microbiome) are hypothesized to contribute to the development of obesity, insulin resistance and type 2 diabetes. Possible mechanisms of action include alteration of the bacterial profile, increased intestinal permeability, and elevated bacterial lipopolysaccharide (LPS) leading to low-grade systemic inflammation termed endotoxemia. The contributions of the human gut microbiome to endotoxemia and skeletal muscle insulin resistance in obesity have not yet been evaluated. We hypothesized that two interventions, sevelamer and Synbiotic, could act in distinct mechanisms on the gut microbiome in obese subjects with insulin resistance to improve skeletal muscle insulin sensitivity and reduce intestinal permeability and endotoxemia. We enrolled 22 lean and 28 obese subjects with normal glucose tolerance who were randomized to receive sevelamer, Synbiotic or placebo daily for 28 days. Insulin sensitivity, intestinal permeability, and body composition were assessed before and after treatment and pre/post plasma, urine, stool and skeletal muscle samples were collected to evaluate intervention effects. Obese subjects had reduced insulin sensitivity compared to lean subjects as assessed by insulin clamp (M/I value, $-30.0 \% \mathrm{p}=0.0023)$. Sevelamer treatment significantly improved insulin sensitivity in obese subjects (M/I value, $+28.5 \%, \mathrm{p}=0.03)$, as well as significantly reduced fasting plasma glucose $(-7.2 \%, p=0.0004)$ and low-density lipoprotein $(-12.8 \%$, $\mathrm{p}=0.0453)$ and tended to reduce CD14+ indicative of attenuated endotoxemia $(-10.1 \%, \mathrm{p}=0.0749)$ (paired T-test). Intestinal permeability and LPS content were not changed by either treatment. Stool microbiota content analysis is ongoing, but preliminary results indicate sevelamer may be a novel intervention for early insulin resistance in humans.

Correspondence to: Eric Baeuerle, Barshop Institute for Longevity and Aging Studies, University of Texas Health Science Center at San Antonio, TX, United States of America.

Email: baeuerle@livemail.uthscsa.edu

Phenylbutyrate: Mechanisms of action and potential to modify resilience in aging
Michael Bene ${ }^{a *}$, Jonathan Dorigatti ${ }^{a}$, Kennedy Mdaki ${ }^{a}$, Kevin Thyne ${ }^{a}$, and Adam Salmon ${ }^{\mathrm{a}}$

${ }^{a}$ University of Texas Health Science Center San Antonio

Phenylbutyrate (PBA) is an FDA-approved drug used for treatment of urea cycle disorders. PBA has been shown to have promiscuous molecular effects that include inhibition of histone deacetylases, modulation of mitochondrial function, and chemical chaperone activity. This spectrum of actions has overlap with multiple potential mechanisms of aging and PBA improves many models of chronic and age-related diseases, including neurodegenerative disorders and metabolic dysfunction. We propose that PBA may be an extremely attractive candidate to address some of the challenges of aging, including loss of resilience, and to gain a better understanding of how such challenges contribute to natural aging and disease development. In our preliminary studies, we have used experiments in cell culture and in vivo mouse studies to characterize the effects of PBA administration. In some cell and tissue types, expression of the redox sensor DJ-1 is increased with PBA; additionally, DJ-1s subcellular localization is altered by drug treatment. Moreover, PBA has significant effects on mitochondrial appearance and dynamics which, together with its effects on DJ-1, imply significant effects on mitochondrial function and bioenergetics which are important cellular processes relevant to aging. We also find PBA administration to alter cellular proteostasis and epigenetics through modification of histone acetylation. Epigenetic changes, alterations to redox environment, mitochondrial dynamics, and ER stress are all found in many aging tissues as well as chronic diseases like Alzheimer's and myopathies such as sporadic-inclusion body myositis. Our planned future studies will address the effect of PBA administration on these outcomes.

Correspondence to: Michael Bene, University of Texas Health Science Center San Antonio.

Emial: bene@livemail.uthscsa.edu

\section{Activation of AMPK by $\beta$-GPA to improve resilience and healthspan in aging mice}

Dorigatti JD ${ }^{\mathrm{a} *}$, Liu $\mathrm{Y}^{\mathrm{a}}$, Thyne $\mathrm{KM}^{\mathrm{a}}$, Mdaki $\mathrm{KS}^{\mathrm{a}}$, Bene MR ${ }^{\mathrm{a}}$, Salmon $\mathrm{AB}^{\mathrm{a}}$ ${ }^{a}$ The Sam and Ann Barshop Institute for Longevity and Aging Studies, The University of Texas Health Science Center at San Antonio, San Antonio TX 78245, USA.

The highly conserved AMP Activated Protein Kinase (AMPK) signaling pathway is partially responsible for maintenance of cellular energy homeostasis and resilience. Under metabolic stress, an increasing AMP:ATP ratio in the cell activates AMPK and its effectors to restore energy balance. $\beta$-Guanidinopropionic acid ( $\beta$-GPA) is a naturally occurring compound that activates AMPK signaling in vivo and improves exercise tolerance, glucose metabolism and mitochondrial metabolism in young rodents. However, it is unknown if $\beta$-GPA may preserve function later in life. Here, we tested how $\beta$-GPA affects physiological markers of age-related decline and cellular 
function in young and old genetically heterogeneous mice and found both age- and sex- specific outcomes. $\beta$-GPA supplementation ( $1 \%$ in food) decreased lean and fat mass in young females but protected old males from latelife declines. Gait performance was improved in $\beta$-GPA treated old males and females, while exercise tolerance test showed increased endurance in the young treatment group. Glycated hemoglobin (HbA1c) levels were lower in young animals and old males fed $\beta$-GPA suggesting improved glucose homeostasis, though old females were unaffected. In muscle, AMPK activation by $\beta$-GPA was blunted in older animals, though we found increased citrate synthase activity indicative of mitochondrial biogenesis. Using high resolution respirometry, we found fatty acid metabolism capacity was elevated in old and reduced in young mice with $\beta$-GPA; this was observed both in vivo and in isolated muscle. These results suggest $\beta$-GPA may improve health and performance in young animals, while also delaying age-related declines when delivered later in life.

Correspondence to: Dorigatti JD, The University of Texas Health Science Center at San Antonio, San Antonio TX 78245, USA.

Emial: dorigatti@livemail.uthscsa.edu

Sex-specific effects of methionine restriction on resilience and health-span

Thyne $\mathrm{KM}^{\mathrm{a} *}$, Liu $\mathrm{Y}^{\mathrm{a}}$, Salmon $\mathrm{AB}^{\mathrm{a}}$

The Sam and Ann Barshop Institute for Aging and Longevity Studies, 15355 Lambda Drive, San Antonio, Texas 78245.

Significant sex differences exist in the response to dietary, genetic, and pharmaceutical interventions that extend lifespan. However, differences between males and females in their response to methionine restriction (MR) have been relatively understudied. MR has also been shown to improve lifespan, improve glucose metabolism, and reduce oxidative stress. Here, we tested whether male and female mice respond similarly to MR in terms of glucose metabolism and markers of health-span. Beginning at 9 months of age, C57BL/6 mice were fed either methionine replete $(0.86 \%$ methionine as total amino acids) or MR $(0.15 \%$ methionine) diets ad libitum. As previously reported, MR reduced body weight and fat mass, and improved glucose metabolism in male mice after 3-month intervention. To our surprise, these results were not replicated in female mice as MR had little to no effect on these parameters relative to mice on methionine replete diet. At the molecular level, MR is known to increase $\mathrm{H} 2 \mathrm{~S}$ production through the Transsulfuration Pathway (TSP). However, sex had no effect on the expression of TSP enzymes in liver. At 24 months of age, males had greater grip-strength, reduced voluntary wheel running, increased $\mathrm{HbAlc}$, and reduced length of time maintained on rota-rod compared to females. However, MR had no effect on most of these parameters even after intervention for $>15$ months. Our data suggest that 1) short-term MR has some sex-specific effects on metabolic function, 2) long-term MR does not significantly delay age-related changes in function across several standard mouse health-span assessments at 24 months.

Correspondence to: Thyne KM, The Sam and Ann Barshop Institute for Aging and Longevity Studies, 15355 Lambda Drive, San Antonio, Texas 78245

Emial: thyne@livemail.uthscsa.edu

Small peptide-based compounds activate the proteasome and attenuate Alzheimer's disease progression and pathology

Maria Gaczynska ${ }^{\mathrm{a}, \mathrm{b}}$, Przemyslaw Karpowicz ${ }^{\mathrm{c}}$, Elzbieta Jankowska ${ }^{\mathrm{c}}$, Jonathan Bohmann $^{\mathrm{d}}$, E. Sandra Chocron ${ }^{\mathrm{a}, \mathrm{b}}$, Erin Munkácsy ${ }^{\mathrm{a}, \mathrm{b}}$, Jodie Cropper $^{\mathrm{a}, \mathrm{b}}$, Andrew M. Pickering ${ }^{\mathrm{a}, \mathrm{b}, \mathrm{e}}$, Pawel A. Osmulski ${ }^{\mathrm{a}, \mathrm{b}}$

a Department of Molecular Medicine, UT Health San Antonio, USA.

${ }^{\mathrm{b}}$ Barshop institute for Longevity and Aging Studies, UT Health San Antonio, USA.

${ }^{c}$ Department of Biomedical Chemistry, Faculty of Chemistry, University of Gdansk, Poland.

${ }^{\mathrm{d}}$ Southwest Research Institute, San Antonio, USA.

'The Glenn Biggs Institute for Alzheimer's \& Neurodegenerative Diseases, UT Health San Antonio, USA

Proteasome is a pivotal enzyme of controlled proteolysis responsible for the catabolic arm of proteostasis. The proteasome-dependent functions include among many others, processes critical for neuronal functions such as synaptic plasticity, vesicle transport, and synaptic signaling. Ominously, proteasome activity is known to be lowered and followed by deregulation of proteasome-related degradation in brains ravaged by Alzheimer's disease (AD). Consequently, deterioration of the proteasomerelated proteostasis would be expected to affect neuronal functions and to drive many of the physiological and symptomatic deficits observed under AD. Thus, augmentation of the proteasome activity may at least prevent the progression of AD. However, since the proteasome is a giant, multifunctional and modular protease challenging to control with synthetic molecules, such a strategy has never been tested. To meet the challenge, we developed a set of proteasome-activating peptidomimetics based on proteasome-binding and blood-brain-barrier-passing fragments of the viral protein HIV-1 Tat. Our compounds enhance the major peptidase activity of the proteasome in vitro by a unique allosteric mechanism. The activation effect is preserved in cellulo and in vivo. The compounds at low-micromolar concentrations reduce cell death in cell lines that either overexpress APP (amyloid precursor protein) or have been treated with $\beta$-amyloid. We have found these peptidomimetics to effectively cross the blood brain barrier in mice and to reduce or even reverse AD-related deficits in learning and memory in fruit fly and mouse models. Here we present selected aspects of design and actions of the compounds.

Correspondence to: Maria Gaczynska, Department of Molecular Medicine, UT Health San Antonio, USA.

Email: gaczynska@uthscsa.edu

LRP1 as a modulator of CXCR4 in hippocampal neurogenesis and neurodegeneration 
Kristi Guerrero a, Swetha Mahesula ${ }^{a}$, Pamela Reed ${ }^{\mathrm{a}}$, Sadiya Ahmad ${ }^{\mathrm{a}}$, Shane Sprague a, Erzsebet Kokovay, PhD ${ }^{\mathrm{a}}$, Naomi L. Sayre, PhD.

${ }^{a}$ University of Texas Health San Antonio, San Antonio, TX.

As the cause of death of a third of senior citizens currently and with prevalence projected to triple by the year 2050 , neurodegenerative diseases pose a growing public health crisis. The urgency of this crisis becomes even more apparent when these statistics are taken in the context of the complete lack of treatments available to stop, slow or prevent disease progression. Our goal is to obtain a better understanding of the disease processes underlying Alzheimer's disease (AD), the most common neurodegenerative disease, so that development of better treatments options is possible. Often the first noticeable symptom in patients with Alzheimer's disease is memory loss, due to early degeneration within the hippocampus. In addition to being integral to learning and memory, the hippocampus is one of only two sites of adult neurogenesis in the brain. Within the sub-granular zone of the dentate gyrus, new neurons are born and integrate into hippocampal circuitry. This neurogenic process is also integral to hippocampal memory functions and can protect from memory loss in AD when preserved. In particular, a chemokine receptor known as CXCR4, plays an important role in guiding the migration of neural stem cells from their niche in the sub-granular zone to integrate into hippocampal circuitry. Loss of CXCR4 activity, either via loss of expression or pharmacologic inhibition, causes hippocampal memory deficits in mouse models. CXCR4 polymorphisms were similarly identified as risk factors for several neurodegenerative diseases in humans. Given this, a better understanding of the regulation of CXCR4 could translate into treatments which inhibit memory loss in AD. Our lab has identified a novel regulator of CXCR4- low density lipoprotein receptor related protein 1 (LRP1). LRP1 itself is implicated in neurodegenerative disease. LRP1 expression is decreased in AD patients. Moreover, LRP1 is a receptor for both ApoE4 and amyloid beta, which each play a significant role in AD pathogenesis. We used a Nestin-Cre inducible mouse model to knock out LRP1 specifically within adult neural stem cells at 2 months of age. Our preliminary data indicate that loss of LRP1 in neural stem cells causes decreased expression of CXCR4, which was associated with migratory deficits in another neurogenic niche, the subventricular zone. Mice with loss of LRP1 in neural stem cells also display memory deficits at 8 months of age, suggesting the hippocampus is also affected. These data suggest that LRP1-mediated positive regulation of CXCR4 in the sub-granular zone is necessary for hippocampal memory function. This hypothesis can be tested using our inducible mouse model, a variety of hippocampal-based functional tests, and in vitro trafficking and expression assays. The research is important because it may elucidate a previously unknown link between three independently identified effectors of neurodegenerative disease and add to a growing knowledge base aimed to furnish disease modulating therapeutics.
Correspondence to : Kristi Guerrero, University of Texas Health San Antonio, San Antonio, TX.

Email: guerrerok@livemail.uthscsa.edu

mTOR promotes BBB breakdown in a model of Alzheimer's disease

Stephen F. Hernandez, Candice E. Van Skike $(\mathrm{PhD})^{\mathrm{a}}$, Nick DeRosa ${ }^{\mathrm{a}}$, Veronica Galvan $(\mathrm{PhD})^{\mathrm{a}, \mathrm{b}, \mathrm{c}}$

${ }^{a}$ Barshop Institute, UT Health San Antonio, Texas.

${ }^{\mathrm{b}}$ Glenn Biggs Institute for Alzheimer's and Neurodegenerative Diseases. ${ }^{c}$ Department of Veterans Affairs South Texas Veterans Health Care System (STVHCS)/GRECC.

Cerebral amyloid angiopathy (CAA) is characterized by fibrillar amyloid $\beta(\mathrm{A} \beta)$ association with cerebrovasculature, which leads to impaired brain vascular function, and is present in $87 \%$ of people with Alzheimer's disease (AD). Previously, it has been shown that inhibition of mTOR by rapamycin prevented vascular leakage in 18 to 19 -month old Tg2576 mice, a mouse model that mimics AD-associated CAA. This finding suggests that mTOR plays a role in regulating the integrity and permeability of the blood brain barrier (BBB). To further expand on this study, the abundance of tight junction proteins, zonula occludens 1 (ZO-1), occludin, and claudin -5 were examined using immunofluorescent confocal microscopy on frozen brain tissue sections of the same $\mathrm{Tg} 2576$ mice from the previous study. Together, these studies demonstrate that attenuation of mTOR by rapamycin preserves BBB integrity by preventing the accumulation of vascular $A \beta$, which also showed a reduced likelihood of cerebral microhemorrhages and an increase in tight junction protein abundance in our transgenic Tg2576 mice. Therefore, these data suggest that mTOR is a key component in vascular $A \beta$ accumulation, BBB breakdown, vascular dysfunction, and BBB permeability in the Tg2576 mice. Thus, the data suggests that using mTOR inhibitors such as rapamycin, an FDA approved drug, may be therapeutic in the pathogenesis of $\mathrm{AD}$ and other dementias with related cerebrovascular dysfunction.

Correspondence: Stephen F. Hernandez, Barshop Institute, UT Health San Antonio, Texas.

Email: hernandezs@uthscsa.edu

Beta2-adrenergic receptor agonists, commonly used as bronchodilators, reduce the risk of Parkinson's disease

MacCarthy $\mathrm{D}^{\mathrm{a}}$, Singh $\mathrm{BB}^{\mathrm{b}}$, Strong $\mathrm{R}^{\mathrm{c}, \mathrm{e}, \mathrm{f}}$, Espinoza $\mathrm{SE}^{\mathrm{d}, \mathrm{e}, \mathrm{f}}$, Wang C-P ${ }^{\mathrm{a}}$, Kamat $\mathrm{A}^{\mathrm{d}, \mathrm{e}, \mathrm{f} *}$

${ }^{a}$ Population Health Sciences, ${ }^{b}$ Dental School, ${ }^{c}$ Pharmacology, ${ }^{\mathrm{d}}$ Medicine, ${ }^{\mathrm{e}}$ Barshop Institute of Longevity and Aging, University of Texas Health at San Antonio and ${ }^{\mathrm{f}}$ South Texas Veterans Health Care System.

Parkinson's disease (PD), is a progressive neurodegenerative disease that is characterized by intraneural accumulation and aggregation of the $\alpha$-synuclein ( $\alpha$-syn) protein, and degeneration of dopaminergic (DA) neurons in the substantia nigra pars compacta $(\mathrm{SNpc})$ region of the brain. Currently, development of new treatments for PD is hindered as the molecular mechanism(s) responsible for PD 
pathogenesis are not fully understood. A recent study for incident $\mathrm{PD}$ in Norway indicated a reduced risk of PD with use of beta-adrenergic receptor subtype ${ }_{2}\left(\beta_{2}-A R\right)$ agonist salbutamol and an increased risk of PD with use of antagonist (beta blocker) propranolol. Beta-adrenergic receptors $\left(\beta_{1}-, \beta_{2^{-}}\right.$and $\beta_{3}$-AR subtypes) mediate the action of catecholamines to modulate important biological responses including bronchodilation and vasodilation. To determine if $\beta_{2}$-AR agonists salbutamol (short-acting) and formoterol (long-acting), which are commonly prescribed bronchodilators for asthma patients, show beneficial effects in a diverse group of patients in US, we conducted studies utilizing the electronic health records (2003-2017) accessed through the VA Corporate Data Warehouse. Cox regression analysis adjusted for covariates and propensity scores for $\beta_{2}$-AR agonist use was utilized to estimate the hazard ratios (HR) of PD associated with $\beta 2-\mathrm{AR}$ agonists (salbutamol, formoterol) relative to those without prescription for salbutamol or formoterol after adjusting for time varying baseline covariates (e.g. age at index date, sex, demographics, and comorbidities) and confounders (adjusted for using inverse propensity scores weighting). The study cohort $(n=1,739,863)$ consisted of 9729 formoterol users, 861 salbutamol users, and 528 patients who had used both formoterol and salbutamol. Our studies indicated that treatment with formoterol and salbutamol reduced the risk of PD [Hazard ratio for salbutamol/formoterol users compared to non-users is $0.514(\mathrm{p}<0.001), 95 \%$ confidence interval $(\mathrm{CI})=0.51-0.53$ for salbutamol and 0.367 $(\mathrm{p}<0.001), 95 \% \mathrm{CI}=0.35-0.39$ for formoterol]. We are currently investigating $\beta_{2}$-AR-mediated mechanisms that are involved in reducing DA neuronal degeneration and $\alpha$-syn aggregation and thus aid in reducing the risk of PD.

Correspondence to: Kamat A, Medicine, Barshop Institute of Longevity and Aging, University of Texas Health at San Antonio and South Texas Veterans Health Care System

Emial: kamat@uthscsa.edu

Evaluation of the aldehyde scavenging agent, hydralazine, in Parkinson's disease.

Anthony Martinez $z^{\mathrm{a}, \mathrm{b}, \mathrm{c} *}$, Vanessa Martinez ${ }^{\mathrm{b}}$, Elizabeth Fernandez ${ }^{\mathrm{a}, \mathrm{b}, \mathrm{d}}$, Randy Strong ${ }^{\mathrm{a}, \mathrm{b}, \mathrm{c}, \mathrm{d}}$

${ }^{a}$ Department of Pharmacology. ${ }^{b}$ Barshop Institute for Longevity and Ag-

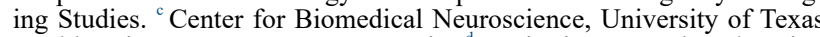
Health Science Center at San Antonio. ${ }^{d}$ Geriatric Research, Education and Clinical Center, South Texas Veterans Health Care Network

Parkinson's disease (PD) affects 1 to $2 \%$ of individuals over the age of 65 years. Consequent to the loss of nigral dopaminergic neurons, reduced striatal dopamine (DA) results in the progressive decline in motor function. A hallmark of familial and sporadic PD is the presence of alpha-synuclein ( $\alpha$ Syn)-rich Lewy bodies in surviving DA neurons. While it remains unclear what mechanism(s) underlies the selective vulnerability of DA neurons, a growing body of evidence implicate the dopamine aldehyde metabolite, 3,4-dihydroxyphenylacetaldehyde (DOPAL). Deletion of the aldehyde dehydrogenase (ALDH) enzymes, Aldh1a1 and Aldh2, result in elevated levels of
DOPAL, reduced tyrosine hydroxylase-immunoreactive neurons, and motor dysfunction. Unpublished data from our lab suggests that the FDA-approved aldehyde scavenging agent, hydralazine, prevents and attenuates motor impairment in Aldh-deficient mice. Additionally, mutations and multiplications in the $\alpha$ Syn gene, SNCA, implicates $\alpha$ Syn in PD. Evidence suggests that DOPAL promotes and stabilizes the formation of pathological $\alpha$ Syn oligomers. The selective generation of DOPAL in DA neurons and its ability to stabilize neurotoxic $\alpha$ Syn oligomers, led us to hypothesize that, $\alpha$ Syn is mechanistically related to the neurochemical and behavioral manifestations of PD consequent to elevated DOPAL. To test this hypothesis, mice deficient in Aldh1a1 and Aldh2 were crossed with mice that overexpress the human wildtype $\alpha$ Syn. We found that overexpression of $\alpha$ Syn in the presence of elevated levels of DOPAL was associated with exacerbation of deficits in motor performance. The administration of hydralazine at $250 \mathrm{mg} / \mathrm{L}$ fails to attenuate motor impairment in mice overexpressing human wildtype $\alpha$ Syn and deficient in Aldh enzymes.

Correspondence to: Anthony Martinez, Center for Biomedical Neuroscience, University of Texas Health Science Center at San Antonio. Email: martinezp3@uthscsa.edu

Funding: VA/BLR\&D (I01 BX001641) and NIH/NIA-Nathan Shock Center of Excellence in Basic Biology of Aging (P30-AG013319)

\section{Exercise Training and Resilience}

\section{Enhancing functional capacity of older veterans with} short session high intensity interval training (HIIT)

Kenneth L. Seldeen ${ }^{\text {* }}$, Ayesha Rahman ${ }^{\mathrm{a}}$, Yonas Redae ${ }^{\mathrm{a}}$, Jeffrey Mador ${ }^{\mathrm{a}}$, Nikhil Satchidanand ${ }^{\mathrm{a}}$, Andrew Ray ${ }^{\mathrm{a}}$, and Bruce R. Troen ${ }^{\mathrm{a}}$

${ }^{a}$ University of Buffalo and Veterans Affairs, Buffalo, NY.

Frailty is a prevalent condition that increases risk for comorbidities, loss of independence, and mortality. Exercise is an effective intervention for frailty, yet participation in older populations is low - as few as $8 \%$ of those 75 and older meet recommendations of 150 minutes/week of moderate intensity exercise. Thus, there is urgent need for new exercise modalities that might appeal to this demographic. Towards this goal we reported that a 3-day-aweek, short session ( $\leq 10$ minutes) high intensity interval training (HIIT) regimen improved muscle mass, physical performance, and reduced frailty in aged mice (from 2428 months of age, human equivalent of $65-75$ years). We now aim to translate our short session HIIT model to older veteran populations including frail, pre-frail, and non-frail males and females between the ages of 65-85. The exercise program for our study includes a 3-minute warm-up at $50 \% \mathrm{VO} 2 \mathrm{max}$, followed by 4 intervals of 1 -minute at $80 \% \mathrm{VO} 2 \mathrm{max}$ interspersed with 1-minute of active recovery using a recumbent exercise bike. Baseline and endpoint readouts include: Frailty, VO2max, regional blood flow, grip and quadriceps strength, short physical performance battery, activities of daily living (ADLs) survey, 
quality of life, enjoyment, and satisfaction questionnaire short form (Q-LES-Q-SF), the physical activity enjoyment scale (PACES) survey, and inflammatory biomarkers (CRP, IL-6 and IL-10). At present our study has reached the halfway point in terms of recruitment and our preliminary findings indicate a 10-minute short-session HIIT exercise protocol improves both strength and endurance in older veterans.

Correspondence to: Kenneth L. Seldeen, University of Buffalo and Veterans Affairs, Buffalo, NY.

Email: seldeen@buffalo.edu

Acknowledgement: Supported by the Department of Veterans Affairs (RX002902) and Indian Trail Foundation.

Cognitive impairment induced by LPS in the rat dentate gyrus is rescued by molecular signaling changes associated with resistance-training exercise

Kerr NR ${ }^{\mathrm{a} *}$, Kelty $\mathrm{TJ}^{\mathrm{a}}$, Childs $\mathrm{TE}^{\mathrm{a}}$, Booth $\mathrm{FW}^{\mathrm{a}}$

${ }^{\mathrm{a}}$ University of Texas Health San Antonio, San Antonio, TX.

Many brain neuroinflammatory diseases lack effective prevention. Mild cognitive impairment (MCI), a forerunner to neuroinflammatory diseases, is ameliorated by resistance-exercise training (RT). The molecular basis by which RT diminishes MCI is understudied. Thus, an experiment was conducted using young female Wistar rats to identify molecular changes in response to RT. MCI was induced via intraventricular lipopolysaccharide (LPS) injection in order to increase inflammation in the dentate gyrus, resulting in a significant increase in TNF- $\alpha$ $(\sim 400 \%)$ and IL-1 $(\sim 1,500 \%)$ mRNA $(\mathrm{P}<0.0001)$ after six weeks. Five days post LPS injection, rats were split into two groups with half performing RT utilizing our ladder climbing model 3 days/week for 6 weeks and half remaining without ladder RT. Six weeks of RT increased lean body mass percentage $(\mathrm{P}<0.05)$, maximum lifting capacity $(\mathrm{P}<0.001)$, and individual muscle masses of the gastrocnemius and tibialis anterior $(\mathrm{P}<0.05)$. Compared to the sedentary controls, the RT group had 1) ameliorated spatial learning deficits $(\mathrm{P}<0.05), 2)$ increased dentate gyrus phosphorylation of IGF-1R, protein kinase B, GSK$3 \beta$ proteins $(\mathrm{P}<0.05)$, components of downstream IGF1 signaling, and 3 ) increased synaptic plasticity marker synapsin protein $(\mathrm{P}<0.05)$ in the dentate gyrus. A follow up experiment was done that showed a significant increase in the phosphorylation of ERK $1 / 2$, GSK-3 $\beta$ proteins and proliferation marker protein PCNA $(\mathrm{P}<0.05)$ twenty-four hours after the third workout in a 1-wk training duration. This data shows that RT ameliorates LPS-induced MCI and could be used as a preventative therapy for aging individuals, especially those with mobility issues.

Correspondence: Kerr NR,University of Texas Health San Antonio, San Antonio, TX.

Email: nrkfkd@mail.uthscsa.edu

\section{Cellular Stress Resilience}

The critical endoplasmic reticulum (ER)-associated protein quality control (ERQC) adaptation of the longlived Caenorhabditis elegans rpn-10(ok1865) proteasomal mutant

Meghna N. Chinchankar ${ }^{\mathrm{a}, \mathrm{b}, \mathrm{c} *}$, Karl A. Rodriguez ${ }^{\mathrm{a}, \mathrm{d}}$, Alfred L. Fisher ${ }^{\mathrm{a}, \mathrm{b}, \mathrm{c}, \mathrm{e}}$ ${ }^{a}$ Barshop Institute for Longevity and Aging Studies, UT Health Science Center at San Antonio (UTHSCSA), San Antonio, TX.

${ }^{\mathrm{b}}$ Center for Healthy Aging, School of Medicine, UTHSCSA, San Antonio, TX.

${ }^{c}$ Division of Geriatrics, Gerontology, and Palliative Medicine, Department of Medicine, UTHSCSA, San Antonio, TX.

${ }^{\mathrm{d}}$ Department of Cell Systems and Anatomy, UTHSCSA, San Antonio, TX.

${ }^{\mathrm{e}}$ Division of Geriatrics, Gerontology, and Palliative Medicine, Department of Medicine, University of Nebraska Medical Center, Omaha, NE.

Protein degradation mechanisms are integral for preserving the proteome. Their reduced efficiency leads to protein aggregation which potentiate several proteotoxic disorders. Paradoxically, we reported that the Caenorhabditis elegans rpn-10(ok1865) proteasome mutant exhibits enhanced proteostasis, elevated stress resistance and extended lifespan. RPN-10/PSMD4 is a ubiquitin receptor on the $26 \mathrm{~S}$ proteasome that targets poly-ubiquitinated substrates to its catalytic core for degradation. Moreover, proteasome dysfunction of the rpn-10 mutant is characterized by reduced, not inhibited, ubiquitin fusion degradation. To elucidate the suite of protective mechanisms, we found that the ER membrane and ER-associated degradation (ERAD) genes were significantly upregulated in the rpn-10 mutant. Thus, we hypothesized that the rpn-10 mutant exhibits enhanced ERQC. Accordingly, the rpn10 mutant exhibits higher ER stress resistance and altered ER homeostasis. Complementarily, attenuated expression of the aggregation-prone $\alpha-1$ antitrypsin (ATZ) reporter proves that ER proteostasis is also ameliorated in the rpn10 mutant. Via a forward genetics screen for suppressors of decreased ATZ aggregation in the rpn-10 mutant, we identified a novel player, H04D03.3, which is a putative homolog of the proteasome-associated adaptor protein ECM29. This finding unexpectedly suggests that the assembly of the rpn-10 mutant proteasome distinctly regulates its ERQC. Further, we observed that cytosolic proteostasis and longevity benefits are contingent on the ER master chaperone hsp-3/-4 (BiP) and ER ATPase cdc-48.2 (p97/VCP), thus highlighting the significance of ERQC in the rpn-10 mutant. Altogether, it appears that mild proteasomal dysfunction induces a unique ERQC adaptation that underlies improved proteostasis and increased longevity of the rpn-10 mutant.

Correspondence to: Meghna N. Chinchankar, Barshop Institute for Longevity and Aging Studies, UT Health Science Center at San Antonio (UTHSCSA), San Antonio, TX.

Email: chinchankar@uthscsa.edu

The protein translation paradox and its implications for the onset of proteostasis collapse and aging

Harper $\operatorname{Kim}^{\mathrm{a}, \mathrm{b}, \mathrm{c}} *$, Andrew Pickering ${ }^{\mathrm{b}, \mathrm{c}}$ 
${ }^{a}$ South Texas Medical Scientist Training Program (STX-MSTP), UT Health San Antonio.

${ }^{\mathrm{b}}$ Department of Molecular Medicine, UT Health San Antonio.

Sam \& Ann Barshop Institute for Longevity and Aging Studies.

High levels of protein translation have been shown to cause age-related functional declines. However, protein translation is high only in young adulthood. Protein translation sharply declines thereafter, staying at a very low level at mid-old ages in many organisms, including humans. These studies collectively raise the protein translation paradox: High protein translation causes agerelated functional declines, but protein translation declines pronouncedly with age. To resolve these contradictory findings, we hypothesize that a rise in protein translation during early adulthood initiates aging at the molecular level by overwhelming protein quality control and triggering disruption of protein homeostasis (proteostasis); consequently, protein translation is repressed with age as an adaptive response to improve proteostasis, slow down aging, and optimize the lifespan. Consistent with the hypothesis, our data suggests that long-lived fly strains (e.g. chico homozygotes) with no signs of early proteostasis collapse, unlike wild types, do not enhance protein translation in early adulthood. To directly test our hypothesis, we transiently modified expression levels of translational machineries in early, late, or whole adulthood of fruit flies via the GeneSwitch (drug-inducible) system. This approach enables us to 1) prevent/enhance a rise in protein translation during early adulthood and 2) block agedependent decline in protein translation. We will examine how these affect life- and health span and levels of polyubiquitinated protein aggregates. Additionally, we will induce early life increase in protein translation in long-lived fly strains and examine whether this abolishes their longlived phenotypes.

Correspondence to: Harper Kim, South Texas Medical Scientist Training Program (STX-MSTP), UT Health San Antonio.

Email: kimsw@livemail.uthscsa.edu

Chronic subordination stress induces tissue-specific cellular senescence in mice

Carey Lyons ${ }^{\mathrm{a}, \mathrm{b} *}$, Maria Razzoli ${ }^{\mathrm{a}}$, Rachel Mansk ${ }^{\mathrm{a}}$, Giada Caviola ${ }^{\mathrm{a}, \mathrm{c}}$, Kohinoor Kahn ${ }^{\mathrm{a}}$, Madeleine Berg ${ }^{\mathrm{a}}$, Judith Campisi ${ }^{\mathrm{de}}$, Jan Van Deursen ${ }^{\mathrm{f}}$, Alessandro Bartolomucci ${ }^{\mathrm{a}}$

${ }^{\mathrm{a}}$ Department of Integrative Physiology and Biology . ${ }^{\mathrm{b}}$ Graduate Program in Neuroscience University of Minnesota, Minneapolis Minnesota. 'Universitá di Parma, Parma Italy. ${ }^{\mathrm{d}}$ Buck Institute for Research on Aging, Novato CA. ${ }^{\mathrm{e}}$ Lawrence Berkeley National Laboratory, Life Sciences Division, Berkeley CA. ${ }^{\mathrm{f}}$ Dept. Biochemistry and Molecular Biology, Mayo Clinic, Rochester MN.

Conventional wisdom posits that severe stress ages a person. Scientific evidence backs this claim, showing that higher perceived stress levels correlate with shorter telomeres, a trigger for cellular senescence. Senescent cells (SNCs) accumulate with age and contribute to the onset and progression of numerous aging-related diseases. Our lab previously showed that lifelong chronic subordination stress (CSS) in mice shortens lifespan and increases se- nescence markers. In this study, we asked whether briefer exposure to mild (restraint) or severe (CSS) stress would be sufficient to increase accumulation of SNCs in young or old mice. We found that in young mice, 4 weeks of CSS, but not restraint, increased expression of senescence markers in a tissue-dependent manner with changes particularly evident in the brain and adipose. Targeted clearance of p16-positive cells in p16-3MR mice normalized expression of senescence and SASP markers, causally linking stress with SNCs accumulation. We also found that while, as expected, natural aging increases expression of senescence markers in the brain, 4 weeks of CSS in old mice did not further augment expression. These results are the first to demonstrate that psychological stress increases abundance of SNCs, implicating them as mediators of stress-induced disease. Our findings mark an important advance toward identifying stress-associated mediators responsible for SNCs accumulation and their impact on aging-related diseases.

Correspondence to: Carey Lyons, Department of Integrative Physiology and Biology, Graduate Program in Neuroscience University of Minnesota, Minneapolis Minnesota.

Email: lyons293@umn.com

Acknowledgements: CL is supported by T32AG029796.

Effects of chronic exposure to aluminum ions on cellular resilience

Kennedy S. Mdaki ${ }^{a *}$, Jonathan Dorigatti ${ }^{\mathrm{a}}$, Kevin Thyne ${ }^{\mathrm{a}}$, Yuhong Liu ${ }^{\mathrm{a}}$, Adam Salmon ${ }^{\mathrm{a}}$

a Sam and Ann Barshop Institute for Longevity and Aging Studies, UT Health at San Antonio, 15355 Lambda Drive, San Antonio, TX 78245.

Introduction. Aluminum (Al) is one of the most abundant metals on earth's crust and chronic exposure leads to measurable accumulation in humans. Al ions $\left(\mathrm{Al}^{3+}\right)$ are a significant cause of oxidative stress generated by mitochondria. Senescent cells are known to have altered mitochondrial bioenergetics, generate oxidative stress, and contribute to chronic inflammation in humans. Accumulation of senescent cells is a significant driving process of aging and aging-related diseases. Aim. We investigated whether chronic exposure to $\mathrm{Al}^{3+}$ promotes the development of cellular senescence. Methods. Using primary fibroblast cells from the skin of young mice, cellular senescence was determined by analyzing the effects of chronic exposure to graded doses of aluminum chloride $\left(\mathrm{AlCl}_{3}\right)$ on cell proliferation, viability, and expression of senescence associated beta-galactosidase(SA $\beta$-gal) using an IncuCyte S3 live cell analysis system. Mitochondrial dynamics were assessed using confocal live cell imaging and western blot analysis. Cellular bioenergetics were assessed using a seahorse XF24. Results. With increasing length of $\mathrm{AlCl}_{3}$ exposure, we found decreased cellular proliferation, increased senescence associated $\beta$-gal, and altered bioenergetics. Expression of p53 (a regulator of senescence) and DRP1 (a regulator of mitochondrial fission) were elevated in the $\mathrm{AlCl}_{3}$ treated cells. Discussion. Our work 
provides vital initial insights onto the potential role of $\mathrm{Al}^{3+}$ on cellular senescence and possible implications on the progression of mammalian aging and disease. Future work will expand the scope of this work by determining the expression of additional markers of senescence, oxidative damage, and mitochondrial dynamics in response to chronic exposure to $\mathrm{Al}^{3+}$ in mammals.

Correspondence to: Kennedy S. Mdaki, Sam and Ann Barshop Institute for Longevity and Aging Studies, UT Health at San Antonio, 15355 Lambda Drive, San Antonio, TX 78245.

Email:mdaki@uthscsa.edu

Tau-induced endothelial cell impairment as a driver of microvascular dysfunction in Alzheimer's disease

Andy Banh ${ }^{\mathrm{a}, \mathrm{b}}$, Stacy Hussong ${ }^{\mathrm{a}, \mathrm{b}}$, Candice Van Skike ${ }^{\mathrm{a}, \mathrm{b}}$, Angela Olson ${ }^{\mathrm{a}, \mathrm{b}}$, Stephen Hernandez ${ }^{\mathrm{a}, \mathrm{b}}$, Rakez Kayed ${ }^{\mathrm{c}}$, Veronica Galvan ${ }^{\mathrm{a}, \mathrm{b}}$

${ }^{a}$ University of Texas Health Science Center at San Antonio. ${ }^{b}$ Barshop Institute for Longevity and Aging Studies. ${ }^{\mathrm{c}}$ University of Texas Medical Branch.

Cerebrovascular dysfunction is the first and most abnormal biomarker in Alzheimer's disease (AD) progression. We showed that pathogenic soluble tau aggregates, which are causally implicated in AD and spread transneuronally, also accumulate in cerebrovascular endothelial cells where they may play a role in AD cerebrovascular dysfunction. Indeed, we found that pathogenic tau impairs endothelium-dependent cerebral blood flow (CBF) responses in P301S(PS19) mice modeling tauopathy. Similar to neuron-to-neuron transmission, soluble tau aggregates were transmitted to human brain microvascular endothelial cells (HBEC), where they decreased microtubule stability and endothelial nitric oxide synthase (eNOS) activation and induced senescence as cycle arrest and the development of a senescence associated secretory phenotype (SASP). Consistent with these observations, microtubule destabilization, eNOS deactivation, and senescence/ SASP were significantly increased in microvasculature of P301S(PS19) mice modeling tauopathy. The mechanisms by which pathogenic tau contributes to cerebrovascular dysfunction in AD, however, are not defined. To answer this question, we measured endothelium-dependent vasodilation in P301S(PS19) mice in which soluble tau aggregates were removed with immunotherapy or remained with control treatment. Removal of soluble tau aggregates was sufficient to restore endothelium-dependent CBF in P301S(PS19) mice. These data indicate that soluble tau aggregates mediate deficits in endotheliumdependent CBF responses in a mouse model of tauopathy and which may be caused by impaired eNOS activation and induction of endothelial cell senescence/SASP. Thus, our studies indicate that soluble tau aggregates mediate cerebrovascular dysfunction in a model of tauopathy and suggest that pathogenic tau removal may hold promise for the treatment of AD and other tauopathies.

Correspondence to: Andy Banh, University of Texas Health Science Center at San Antonio.

Email: banh@livemail.uthscsa.edu
Tau-induced astrocyte senescence: A novel mechanism for neural dysfunction in Alzheimer's disease.

Angela Olson $^{\mathrm{a}, \mathrm{b} *}$, Stacy Hussong ${ }^{\mathrm{a}, \mathrm{b}, \mathrm{c}}$, Aubrey Sills ${ }^{\mathrm{b}}$, Rakez Kayed ${ }^{\mathrm{f}}$, Veronica Galvan ${ }^{\mathrm{a}, \mathrm{b}, \mathrm{c}}$.

${ }^{a}$ Department of Cellular \& Integrative Physiology, University of Texas Health at San Antonio, TX, USA.

${ }^{\mathrm{b}}$ Barshop Institute for Longevity and Aging Studies, University of Texas Health at San Antonio, TX, USA.

${ }^{c}$ South Texas Veterans Heath Care System, San Antonio, TX, USA. 4Department of Neurology, University of Texas Medical Branch at Galveston, TX USA.

Chronic sterile inflammation is a pathological feature of Alzheimer's disease (AD) and other neurodegenerative diseases. The mechanisms that drive neuroinflammation and its impact on AD progression are still incompletely understood. The accumulation of molecular damage in somatic cells can trigger cellular senescence, an irreversible state of cell cycle arrest accompanied by the expression of proinflammatory mediators known collectively as the "senescence-associated secretory phenotype" (SASP). Misfolded tau forms pathogenic soluble aggregates that are released extracellularly and are transmitted trans-neuronally, promoting native tau phosphorylation and aggregation in target cells. We recently showed that, in addition to neurons, pathogenic soluble extracellular tau aggregates propagate to brain microvascular endothelial cells, where microtubule destabilization triggers senescence/SASP. Because astrocytes have a critical role in the regulation of both synaptic function and cerebral blood flow and are directly exposed to tau at its site of release at the tripartite synapse, we conducted studies to define whether soluble aggregated tau propagates to astrocytes, inducing astrocyte senescence/SASP and neuronal dysfunction/damage. Our studies indicate that tau can be propagated transcellularly to astrocytes, triggering cellular senescence/SASP. Our studies suggest that astrocyte senescence is detrimental to dendritic and synaptic structure and density, suggesting that pathogenic soluble tau-induced astrocyte senescence may contribute to synaptic dysfunction and loss in AD. Drugs that eliminate senescent cells are FDA-approved and antibody-based approaches to remove tau from brain are already in clinical trials. Our studies suggest that these interventions could be effective in the treatment of $\mathrm{AD}$ and other tauopathies.

Correspondence to: Angela Olson, Department of Cellular \& Integrative Physiology, University of Texas Health at San Antonio, TX, USA.

Email: olsona@livemail.uthscsa.edu

Oligomeric tau contributes to cerebrovascular deficits through nitric oxide synthase dysfunction

Candice E. Van Skike, Ph.D. ${ }^{\text {** }}$, Andy Banh, B.S. ${ }^{\text {a }}$, Stacy A. Hussong, Ph.D. ${ }^{a}$, and Veronica Galvan, Ph.D. ${ }^{a}$

${ }^{a}$ University of Texas Health San Antonio, San Antonio, TX.

Brain microvascular deficits were recently identified as the earliest and most abnormal biomarker in AD progression. We recently reported the accumulation of pathogenic 
soluble tau aggregates (oligomers) in cerebromicrovasculature of $\mathrm{AD}$ and other tauopathies. The impact of cerebrovascular accumulation of pathogenic tau, however, is not yet understood. To define the functional consequences of cerebrovascular tau we used laser Doppler flowmetry to assess microvascular function as (a) the coupling of cerebral blood flow (CBF) to neuronal activity (neurovascular coupling, NVC) and (b) endothelium-dependent vasodilation in mice modeling tauopathy where pathogenic tau was removed with immunotherapy, or remained in brain. Changes in CBF in barrel cortex as a result of whisker stimulation or topical acetylcholine application provided measures of NVC and endothelium-dependent vascular reactivity respectively. The contributions of neuronal (nNOS) and endothelial (eNOS) nitric oxide synthases to $\mathrm{NVC}$ were determined through the use of nNOS-selective and general NOS inhibitors. Our data show that isolated overexpression of mutant human tau in the P301S(PS19) model of tauopathy is sufficient to impair barrel cortex $\mathrm{NVC}$, and that this deficit is mediated by a profound reduction of nNOS activity. Tauopathy in P301S(PS19) mice also impaired eNOS-dependent cortical vasoreactivity, a deficiency that was mitigated by removal of pathogenic tau aggregates with immunotherapy. Our data suggest that the inhibition of NOS activation by pathogenic soluble tau aggregates may underlie microvascular dysfunction in tauopathies, including AD. Therapeutic removal of pathogenic tau may be used to delay disease onset and/or slow progression of $\mathrm{AD}$ and potentially other tauopathies.

Correspondence to: Candice E. Van Skike, University of Texas Health San Antonio, San Antonio, TX.

Email: vanskike@uthscsa.edu

Deficits in nonsense-mediated RNA decay contribute to increased translation and neuronal death in tauopathy

Gabrielle Zuniga ${ }^{a}$, Bess Frost ${ }^{\mathrm{a}}$

${ }^{a}$ University of Texas Health San Antonio, Cell Systems and Anatomy, San Antonio, TX

Cells utilize the nonsense mediated RNA decay (NMD) pathway for post-transcriptional control of gene expression and degradation of aberrant RNA that could otherwise encode potentially toxic proteins. We have recently reported that transgenic expression of pathogenic tau, a protein that accumulates in Alzheimer's disease and related "tauopathies," promotes neurodegeneration by increased nuclear export of polyadenylated RNA through nuclear envelope invaginations in Drosophila and human tauopathy. Nuclear pores line these invaginations and facilitate rapid nuclear export of polyadenylated RNA in Drosophila and human tauopathy. Increased mRNA export may lead to increased cytoplasmic RNA that overwhelms the ability of NMD factors to degrade aberrant or error-containing RNA. We find that genetic manipulation of NMD machinery, but not other RNA quality control pathways, significantly modifies tau-induced neurotoxicity. In addition, the ability of NMD to clear aberrant RNA transcripts was shown to decrease with age and increase after genetic manipulation of RNA export factors in control and tau transgenic Drosophila. These results are consistent with the finding that global translation is increased in adult tau transgenic Drosophila and Drosophila with RNAi-mediated knockdown of core NMD factors. Our preliminary studies suggest a necessary role of NMD in post-transcriptional control of gene expression by preventing accumulation of aberrant RNA transcripts and translation into proteins that are toxic to neurons in tauopathy.

Correspondence to: Gabrielle Zuniga, University of Texas Health San Antonio, Cell Systems and Anatomy, San Antonio, TX.

Email: zunigag3@livemail.uthscsa.edu

Deficiency of a novel depalmitoylase exacerbates dietinduced obesity, insulin resistance, and inflammation

Jason Hadley B.S. ${ }^{\text {a* }}$, Jiyoon Ryu Ph.D. ${ }^{\text {a }}$, Lily Dong Ph.D. ${ }^{a}$

${ }^{a}$ University of Texas Health San Antonio, San Antonio, TX.

Protein palmitoylation is a reversible post-translational modification that has gained rapid attention for its ability to modulate a diverse range of signaling pathways in cells. Depalmitoylases are a class of enzymes that catalyze the removal of this lipid group from target proteins, which subsequently alters the subcellular localization of their target molecules. Recently, we identified LF8 as a potential novel depalmitoylase and found its transcriptional levels are positively associated with insulin sensitivity in mouse liver. Since LF8 is uncharacterized in mammals, we have generated LF8 whole body $\mathrm{KO}$ mice to test the physiological function. Deletion of the LF8 gene in mice leads to an exacerbated high fat diet-induced obesity phenotype and pronounced hepatic inflammation. Interestingly, we found LF8 expression to be largely restricted to peritoneal macrophages and its loss results in aberrant cytokine signaling. These results not only reveal an important role of depalmitoylation in regulating insulin sensitivity and diet-induced chronic inflammation, but also suggest that regulation of peritoneal macrophage function contributes to systematic energy homeostasis.

Correspondence to: Jason Hadley B.S., University of Texas Health San Antonio, San Antonio, TX.

Email: hadleyj@uthscsa.edu

Loss of vacuolar acidity results in iron sulfur cluster defects and divergent homeostatic responses during aging in Saccharomyces cerevisiae

Kenneth L. Chen ${ }^{\mathrm{a}}$, Kathleen L. Helge ${ }^{\mathrm{b}}$, Matt Kaeberlein ${ }^{\mathrm{a}}$, Brian M. Was$\mathrm{ko}^{\mathrm{b} *}$

${ }^{\mathrm{a}}$ Department of Pathology, University of Washington, Seattle, WA, USA 98195.

${ }^{\mathrm{b}}$ Department of Biology and Biotechnology, University of HoustonClear Lake, Houston, TX USA 77058.

The loss of vacuolar/lysosomal acidity is an early event during aging that has been linked to

mitochondrial dysfunction. However, it is unclear how loss of vacuolar acidity results in age-related dysfunction. 
Through unbiased genetic screens, we determined that increased iron uptake can suppress the mitochondrial respiratory deficiency phenotype of yeast vma mutants, which have lost vacuolar acidity due to genetic disruption of the vacuolar ATPase proton pump. Yeast vma mutants exhibited nuclear localization of Aft1, which turns on the iron regulon in response to iron sulfur cluster (ISC) deficiency. This led us to find that loss of vacuolar acidity with age in wildtype yeast causes ISC defects and a DNA damage response. Using microfluidics to investigate aging at the single cell level, we observe grossly divergent trajectories of iron homeostasis within an isogenic and environmentally homogeneous population. One subpopulation of cells fails to mount the expected compensatory iron regulon gene expression program and suffers progressively severe ISC deficiency with little to no activation of the iron regulon. In contrast, other cells show robust iron regulon activity with limited ISC deficiency, which allows extended passage and survival through a period of genomic instability during aging. These divergent trajectories suggest that iron regulation and ISC homeostasis represent a possible target for aging interventions.

Correspondence to: Brian M. Wasko, Department of Biology and Biotechnology, University of Houston-Clear Lake, Houston, TX USA 77058 Email: wasko@uhcl.edu

\section{The aging immune system drives senescence and dys-} function of peripheral tissues

Matthew J. Yousefzadeh ${ }^{\mathrm{a}, \mathrm{b}, \mathrm{c}}$, Rafael R. Flores ${ }^{\mathrm{a}, \mathrm{b}, \mathrm{c}}$, Yuxiang Cui ${ }^{\mathrm{d}}$, Robert W. Brooks ${ }^{\text {a }}$, Jing Zhao ${ }^{\text {a }}$, Luise A. Angelini ${ }^{\mathrm{a}, \mathrm{b}, \mathrm{c}}$, Erin A. Wade ${ }^{\mathrm{a}}$, Christy E. Trussoni ${ }^{\mathrm{e}}$, Jonathan I. Kato ${ }^{\mathrm{a}}$, Collin A. McGuckian ${ }^{\mathrm{a}, \mathrm{b}, \mathrm{c}}$, Dong Wang ${ }^{\mathrm{f}}$, Qing Dong $^{\mathrm{f}}$, Prashanti Patil ${ }^{\mathrm{f}}$, Ken Li ${ }^{\mathrm{f}}$, Aiping Lu ${ }^{\mathrm{g}, \mathrm{h}}$, Ryan D. O’Kelly ${ }^{\mathrm{a}, \mathrm{b}, \mathrm{c}}$, Warren C. Ladiges ${ }^{i}$, Christin E. Burd', Nicholas F. LaRusso ${ }^{\text {e }}$, Smitha P.S. Pillai $^{\mathrm{k}}$, Nam V. Vo ${ }^{\mathrm{f}}$, Eric E. Kelley ${ }^{1}$, Yinsheng Wang ${ }^{\mathrm{d}}$, Johnny Huard ${ }^{\mathrm{g}, \mathrm{h}}$, Paul D. Robbins ${ }^{\mathrm{a}, \mathrm{b}, \mathrm{c}} *$, Laura J. Niedernhofer, ${ }^{\mathrm{a}, \mathrm{b}, \mathrm{c} *}$

${ }^{a}$ Institute on the Biology of Aging and Metabolism, University of Minnesota. ${ }^{b}$ Department of Biochemistry, Molecular Biology and Biophysics, University of Minnesota. ${ }^{\mathrm{c}}$ Department of Molecular Medicine and the Center on Aging, The Scripps Research Institute ${ }^{\mathrm{d}}$ Department of Chemistry, University of California Riverside. ${ }^{e}$ Division of Gastroenterology and Center for Cell Signaling in Gastroenterology. ${ }^{\mathrm{f}}$ Department of Orthopedic Surgery, University of Pittsburgh. ${ }^{\mathrm{g}}$ Steadman Philippon Research Institute. ${ }^{\mathrm{h}}$ Department of Orthopedic Surgery, McGovern Medical School, University of Texas Health Science Center at Houston. ${ }^{i}$ Department of Comparative Medicine, University of Washington. ${ }^{j}$ Departments of Molecular Genetics and Cancer Biology and Genetics, The Ohio State University. ${ }^{k}$ Fred Hutchinson Cancer Research Center, Seattle. ${ }^{1}$ Department of Physiology \& Pharmacology, West Virginia University

Aging of the immune system, or immunosenescence, prevents the elderly from mounting a productive immune response against pathogens or to vaccinations, contributing to infections that are common causes of death in the elderly. To define the contribution of immune aging to organism aging, Ercc1, which encodes a subunit of the DNA repair endonuclease ERCC1-XPF, was deleted only in hematopoietic cells of the mouse to increase endogenous DNA damage and oxidative stress. VaviCre+/-;Ercc1-/fl mice were healthy into adulthood, but then displayed both attrition and senescence of immune cell populations. The mice had accelerated, progressive onset of immunosenes- cence with reduced T, B and NK cell function. Remarkably, non-lymphoid organs also had increased senescence and oxidative stress, resulting in premature aging of liver, kidney and intervertebral disc and reduced repair capacity of injured muscle, indicating that senescent, aged immune cells contribute to systemic aging. Indeed, transplantation of splenocytes from Vav-iCre+/-;Ercc1-/fl or old wildtype mice induced senescence in multiple tissues in trans. Conversely, transplantation of immune cells from young mice into aged animals reduced the senescent cell burden in multiple tissues. Short-term treatment of VaviCre+/;Ercc1-/fl mice with rapamycin improved $\mathrm{T}$ and $\mathrm{B}$ cell function, demonstrating that immunosenescence is malleable. Taken together, these data demonstrate that an aged immune system contributes to driving systemic aging and represents a key therapeutic target to extend healthy aging.

Correspondence to: Matthew J. Yousefzadeh, Institute on the Biology of Aging and Metabolism, University of Minnesota.

Email:myousefz@umn.edu

Funding: This work was supported by the NIH grants P01 AG043376, U19 AG056278, R56 AG059676, K26 AG001057 and R01 AG044376, Glenn Foundation and the Irene Diamond/AFAR.

Identification of a novel adipokine tetranectin and its metabolic function

George Plasko ${ }^{a *}$, Sija He ${ }^{a}$, Jingjing Zhang ${ }^{b}$, Fen Liu ${ }^{b}$, Juli Baia, ${ }^{a, b}$ Lily Dong $^{\mathrm{c}}$, Feng Liu ${ }^{\text {a, }}$

${ }^{a}$ Department of Pharmacology, University of Texas Health at San Antonio, San Antonio, TX 78229

${ }^{\mathrm{b}}$ Department of Metabolism and Endocrinology, Metabolic Syndrome Research Center, Second Xiangya Hospital, Central South University, Changsha, Hunan 410011, China.

${ }^{\mathrm{c}}$ Department of Cell Systems \& Anatomy, University of Texas Health at San Antonio, San Antonio, TX 78229.

Obesity has an established association with insulin resistance, type 2 diabetes mellitus, dyslipidemia, and atherosclerosis, with a heavy burden on the healthcare system. Preventing the progression from obesity to insulin resistance requires an understanding of the regulatory mechanisms involved in the loss of insulin sensitivity. Adipose tissue is well known to function as an endocrine organ that produces many kinds of adipokines, such as leptin, adiponectin, resistin, and retinol-binding protein 4 etc. In the current study, we report the identification and initial characterization of a novel adipokine tetranectin, the C-type lectin domain family 3 member B (CLEC3B) gene coded protein that is highly enriched in white adipose tissue. We found that the serum level of tetranectin was much higher in both obese and diabetic patients. Knocking out the tetranectin gene in mice protected against glucose intolerance in males but reduced insulin and glucose tolerance in females, without effects on food intake and body weight for either sex. Mechanistically, tetranectin targets liver tissues and its deficiency improves insulin-stimulated AKT phosphorylation at serine 473 . Taken together, we have identified a novel adipokine which mediates metabolic crosstalk among tissues to maintain systemic glucose me- 
tabolism. Further investigation of tetranectin's function could yield a new target for the development of effective therapeutic treatment for obesity and its associated metabolic diseases.

Correspondence: George Plasko, Department of Pharmacology, University of Texas Health at San Antonio, San Antonio, TX 78229.

Email: plasko@livemail.uthscsa.edu

\section{Nonhuman Primate Models of Resilience}

Monkeying with resilience: The marmoset as a model for pre-clinical intervention.

Aubrey Sills ${ }^{\mathrm{a}}$, Joselyn Artavia ${ }^{\mathrm{a}}$, Adam Salmon ${ }^{\mathrm{a} *}$

${ }^{\text {a }}$ Barshop Institute, UT Health San Antonio.

The challenge to recover from physiological issues that were once relatively easy to do in young age becomes increasingly more difficult with age. Effective models of resilience will improve the translation of interventions that address resilience in clinical application. While common animal models (e.g., rodents) have advantages in preclinical development, testing in nonhuman primate models may offer advantages as pre-clinical models of translation due to greater similarities in basic physiological functions with humans. Common marmosets are small, New World monkeys with a relatively short lifespan and small size compared to other primates. We have utilized marmosets as a pre-clinical nonhuman primate model of interventional geroscience as well to develop, through reverse translation, useful models of human aging pathology. We are identifying several potential markers of resilience in aging marmosets that might have clinical relevance in terms of interventions. Guided by established rodent and human frailty indices, we have developed a marmoset accumulation of deficits index that includes objective (body weight, medical records, etc.) and subjective assessments of functional deficits. In addition, we find specific hematological markers in aging marmosets that may be used to define overall resilience within the context of clinical relevance. Lastly, we are developing cellular resilience as a means to define biological resilience at an individual level using fibroblast cell lines derived from the skin of aging animals. Overall, defining the effect of age on resilience in the marmoset may aid in developing geroscience-based approaches to clinical intervention.

Correspondence to: Adam Salmon, Barshop Institute, UT Health San Antonio.

Email: salmona@uthscsa.edu

Left ventricular remodeling proceeds from young adulthood into midlife in intrauterine growth restricted baboons

Clarke GD ${ }^{\mathrm{a}} *$, Huber $\mathrm{H}^{\mathrm{b}}$, Li C $\mathrm{C}^{\mathrm{b}}$, Kuo $\mathrm{AH}^{\mathrm{c}}$, Nathanielsz $\mathrm{PW}^{\mathrm{b}}$

${ }^{a}$ UT Health Science Center San Antonio.

${ }^{\mathrm{b}}$ University of Wyoming.
${ }^{\mathrm{c}}$ Massachusetts General Hospital.

Previous cross-sectional studies have shown young adult baboons ( 5-6 y.o.) subjected to intrauterine growth restriction (IUGR), by maternal calorie restriction during pregnancy and lactation, exhibit ventricular remodeling with mildly impaired heart function relative to age/sex matched controls (CTL). The present study was designed to assess the degree of progression of this condition. METHODS: In this longitudinal study, cardiac MRI was performed on male IUGR baboons $(n=7)$. A 3 Tesla, Siemens MRI system was used with phased array coils, parallel imaging acquisition and breath hold during the scan. Studies of IUGR animals occurred at $4.7+0.1 \mathrm{yr}$ intervals; the first scan was at $5.8+1.2$ y (human equivalent 24 years) and the second (scan 2) at 10.4+1.2 yr (human equivalent 40 years). Scan on the CTL animals $(\mathrm{N}=4)$ occurred at $5.3+1.4$ years. RESULTS: Change in body weight over 4.7 years was less in the IUGR group $(\Delta \mathrm{wt}=6.3+6.1 \mathrm{~kg})$ than in the CTL group $(\Delta \mathrm{wt}=11.5+8.2$ $\mathrm{kg}$ ). Left ventricular (LV) ejection fraction (EF) was significantly greater in IUGR animals in scan $2(+10.7 \%$, $\mathrm{p}=0.03)$ but not in normal controls $(+1.8 \%)$. Stroke volume and end-diastolic LV volume were normalized to body surface area (BSA). SV/BSA (17.6+4.9, 31.5+12.3 $\mathrm{mL} \mathrm{m}-2 ; \mathrm{p}=0.016)$ and EDV/BSA $(47.3+13.6,64.5+18.8$ $\mathrm{mL} \mathrm{m}-2 ; \mathrm{p}=0.045)$ also were significantly increased in IUGR animals but not in controls. In IUGR subjects, $\Delta w t$ was significantly and positively correlated with $\Delta \mathrm{EF}$ $(\mathrm{r}=0.86 . \mathrm{p}=0.01)$. CONCLUSIONS: In IUGR, but not in CTL baboons, cardiac function adaptations continue into midlife and are related to increases in body weight with aging. We conclude that IUGR programs cardiovascular function and that programmed change continue into midlife.

Correspondence to: Clarke GD, UT Health Science Center San Antonio. Email: clarkeg@uthscsa.edu

The impact of aging on vaccine-induced memory recall responses

Julia M. Scordo ${ }^{\text {** }}$, Tucker J. Piergallini ${ }^{a}$, Colwyn Headley ${ }^{\mathrm{a}}$, Ron E. Banks $^{b}$, James F. Papin ${ }^{b}$, and Joanne Turner ${ }^{a}$

${ }^{a}$ Texas Biomedical Research Institute, San Antonio, TX.

${ }^{\mathrm{b}}$ Division of Comparative Medicine, The University of Oklahoma Health Sciences Center, Oklahoma City, OK.

By 2035, elderly individuals will outnumber children for the first time in US history resulting in substantial public health and financial impact. As we age, so does our immune system, increasing our susceptibility to infections. Vaccinations are a vital tool to combat infections, yet they are much less effective in the elderly. Improving health outcomes in our aging population requires a better understanding of how the aging immune system impacts vaccine efficacy. To do this we are using the classical delayed-type hypersensitivity model of Mycobacterium bovis BCG vaccination and tuberculin skin test (TST). In this model, BCG vaccination generates BCG-specific 
immune cells that are recruited to the skin upon TST challenge. In our studies we BCG-vaccinate adult and elderly baboons and perform skin challenges eight weeks after vaccination by TST. Three days and one week after challenge we perform skin biopsies to examine BCG-specific cellular memory responses in the skin. We also determine BCG-specific memory responses in the blood to compare tissue and systemic responses within each vaccinated animal. We have shown that Mycobacterium tuberculosis (M.tb)-specific $\mathrm{T}$ cell responses are not altered in the blood of M.tb-infected aged individuals, suggesting that impaired immunity occurs in the tissue. Our data is supported by published literature showing elderly TST responses are decreased. We expect aged BCG-vaccinated baboons will have decreased and delayed vaccine-induced memory responses in the skin due to age-associated alterations, such as pro-inflammatory and oxidative factors, that hinder $\mathrm{T}$ cell migration to and function in the tissue.

Correspondence to: Julia M. Scordo, Texas Biomedical Research Institute, San Antonio, TX.

Email: jscordo@txbiomed.org

\section{Novel Procedures to Support Resilience Studies}

Transcriptomic analysis reveals upregulated microglia phagocytotic signals in the aging sub-ventricular zone

Ronald Cutler $^{\mathrm{a} *}$, Erzsebet Kokovaya ${ }^{\mathrm{b}}$

${ }^{a}$ Department of Cell Systems and Anatomy, University of Texas Health Science Center at San Antonio, San Antonio, TX 78229, United States. ${ }^{\mathrm{b}}$ The Barshop Institute on Longevity and Aging Studies, University of Texas Health Science Center at San Antonio, San Antonio, TX 78229, United States.

The neural stem cell (NSC) pool in the subventricular zone (SVZ) experiences significant losses in number as well as proliferative capacity during aging, which functionally results in reduced cognition and neural plasticity. Microglia (MG) provide support for neurogenesis in the young SVZ while recent evidence from our lab indicates that during aging, MG attain an activated/pro-inflammatory phenotype that has been shown to contribute to agedependent losses in neurogenesis. However, the decline in the NSC pool remains to be fully elucidated and evidence of apoptosis to account for the loss of NSCs during aging is limited. We hypothesize that the aged SVZ MG phenotype may be indicative of increased phagocytic activity against NSCs and thus contributes to the decline of the NSC pool with aging. Here, we re-analyzed RNAsequencing data from multiple studies of the aging SVZ to test if the phagocytic transcriptional program is upregulated in aged MG or whether "eat-me" signals are upregulated in aged NSCs. We found significant upregulation of genes within the complement system (e.g. C1qa, C1qb, C3) in both aged NSCs and aged MGs, Trem2 receptor in aged MG, and scavenger receptor (e.g. Scarb1, Fcrls, Scarf2) in MG. Gene ontology (GO) analysis of upregulated genes revealed top enrichments in 'innate immune response' and 'response to interferon-beta' in aged NSCs and MGs where interferon-beta is known to activate phagocytic activity. However, almost all purinergic receptor related genes (e.g. P2ry12-13, P2rx7, P2ry2/6) were downregulated in aged MGs. Using network analysis, CXCL13 was identified to be highly upregulated during aging and at the intersection of the majority of GO processes. Soluble bridging molecules that are displayed on apoptotic cells such as CALR, Gas6, PROS1, and Tub were upregulated in aged NSCs. But, phosphatidylserine receptor expression, an anchored "eat-me" signal, was not detected in aged NSCs. Lastly, due to an enrichment of interferon signatures, transposon expression was examined which found over 500 differentially expressed transposons in aged NSCs and MGs. While phagocytosis signaling is complex and experimental validation is warranted, this transcriptomic study suggest that MG may indeed be more phagocytic in the aging SVZ.

Correspondence to: Ronald Cutler, Department of Cell Systems and Anatomy, University of Texas Health Science Center at San Antonio, San Antonio, TX 78229, United States.

Email: cutlerr@1ivemail.uthscsa.edu

\section{A facile radiosynthesis and validation of novel tau PET tracer [F-18]-MK-6240}

Ramesh Neelamegam ${ }^{\mathrm{a}, \mathrm{b}}$, , Daniel Yokell ${ }^{\mathrm{b}}$, Georges El Fakhri

${ }^{a}$ Department of Radiology, University of Texas Health Science Center San Antonio, Research Imaging Institute, Glenn Biggs Institute for Alzheimer's and Neurodegenerative Diseases, San Antonio, Texas.

${ }^{\mathrm{b}}$ Gordon Center for Medical Imaging Massachusetts General Hospital, Harvard Medical School Boston MA United States.

[18F]MK-6240, a novel pyridine isoquinoline amine derivative developed by Merck, showed a high sensitivity and specificity for PHF-tau binding. In autoradiographic studies, MK-6240 showed high affinity to tangle-rich AD brain homogenates and large amounts of displaceable binding in the gray matter of $\mathrm{AD}$ brain sections. Absence of off-target binding of [18F]MK-6240 to MAO-A and MAO-B was confirmed in preclinical studies as well. Recent clinical studies have demonstrated that spatial patterns of MK-6240 binding were consistent with neuropathological staging of NFTs. Unlike flortaucipir ([18F] T807) and [18F]THK5351, off-target binding of [18F] MK-6240 was not observed in the basal ganglia and choroid plexus although mild tracer retention was observed in the substantia nigra and meninges. A novel tracer [18F] PI-2620 has also shown a lack of off target binding in the basal ganglia. Intriguingly, a preclinical analysis has suggested a high-affinity binding of [18F]PI-2620 to PSP and Pick's disease brains. To confirm these initial observations, several clinical studies are ongoing in non- $\mathrm{AD}$ patients. [18F]MK-6240, a novel tau positron emission tomography (PET) tracer recently discovered for the in vivo detection of neurofibrillary tangles, has the potential to improve diagnostic accuracy in the detection of Alzheimer disease. Herein, we report the comparison of [18F]MK-6240 radiochemical yields with different F-18 
elution reagents and different temperatures. Nucleophilic fluorination of the 5-diBoc-6-nitro precursor with potassium cryptand $[18 \mathrm{~F}]$ fluoride $(\mathrm{K}[18 \mathrm{~F}] / \mathrm{K} 222)$ or tetraethylammonium [18F]fluoride (TEA[18F]) were performed by conventional heating, followed by thermal N-diBoc deprotection. Then radiochemical conversion (RCC) of the measured by radio-TLC. The crude product was purified by using ethanol and sodium acetate solution and further diluted with phosphate buffered saline then sterile filtered to obtain a solution suitable for injection. Results: HPLC analysis of formulated product revealed high radiochemical $(>98 \%)$ and high chemical purity. Formulated [18F] MK-6240 maintained stability, as measured by radioHPLC and TLC, and met specifications for visual inspection and $\mathrm{pH}$ over a period of 6 hours. This work presents a novel automated radiosynthesis methods for [18F]MK6240 which eliminates the need for the presence of acid for deprotection of $\mathrm{N}$-diBoc groups which makes the synthesis feasible on the Tracerlab FX2 N and other automated synthesis modules. It also eliminates the need for solid phase extraction (SPE) re-formulation of the purified product. The methodology described herein can facilitate multi-center trials and widespread use of this radiopharmaceutical for imaging tau protein in vivo.

Acknowledgements. We thank the Alzheimer's Drug Discovery Foundation for generously providing funding for this research. We also thank David F. Lee Jr., Steven Gallo and Tim Beaudoin of the Massachusetts General Hospital for isotope production and technical support.

Correspondence to: Ramesh Neelamegam, Department of Radiology, University of Texas Health Science Center San Antonio, Research Imaging Institute, Glenn Biggs Institute for Alzheimer's and Neurodegenerative Diseases, San Antonio, Texas

Email: neelamegam@uthscsa.edu

Optimization of $\mathrm{C}$. elegans protein extraction method for proteasome kinetic assay and western blot analysis
Jessica L. Scheirer, ${ }^{\mathrm{a}, \mathrm{b}}$, Meghna N. Chinchankar ${ }^{\mathrm{a}, \mathrm{b}}$, Alfred L. Fisher ${ }^{\mathrm{c}}$, Karl A. Rodriguez $z^{\mathrm{a}, \mathrm{b}}$

${ }^{a}$ Sam and Ann Barshop Center for Longevity and Aging Studies, University of Texas Health Science Center San Antonio (UTHSCSA), San Antonio, TX

${ }^{\mathrm{b}}$ Department of Cell Systems and Anatomy, UTHSCSA.

${ }^{\mathrm{c}}$ Division of Geriatrics, Gerontology, and Palliative Medicine, Department of Medicine, University of Nebraska Medical Center.

Accumulation of protein aggregates are a common pathology in many neurodegenerative disorders. Cellular maintenance of the proteome is overseen by a complex system of cellular pathways and molecular components. One of the ways cells remove protein aggregates is by utilizing the ubiquitin proteasome system (UPS). Concomitantly, proteasome activity declines with age leading to aggregation accumulation, which may be due to a function decline in the overall protein homeostasis network known to occur during the aging process. Consequently, investigating proteasome activity via kinetic based assays has become a regular method used in multiple laboratories. Caenorhabditis elegans is an animal model that has been used in varying fields of study such as neurobiology, genetics, developmental biology, aging and more recently biomedical and environmental toxicology, due to its wellcharacterized genome, short lifespan, large brood size, and $83 \%$ of its proteome is homologous to human genes. One drawback is C. elegans cuticle, an immensely elastic and durable exoskeleton, making protein extraction arduous and requiring large number of animals to achieve the necessary concentrations required for analysis. To overcome this obstacle, we have optimized a protein extraction method for C. elegans utilizing a small sample size for use in the kinetic proteasome assay and western blot analysis.

Correspondence to: Karl A. Rodriguez, Department of Cell Systems and Anatomy, UTHSCSA.

Email: RodriguezK@uthscsa.edu

Cite this article as: Ladiges W, Ikeno Y, Wang L, editors. Resilience in aging and age-related disease. Abstracts from the 2019 Barshop Conference on Aging; 2019 Oct 11-13; Mayan Dude Ranch in Bandera, TX, USA. Ant publishing; 2020, 2(1): 01-13. 